\title{
Subjetivación femenina: una mirada a la niña que me ronda*
}

\author{
Female Subjectivity: A Look at the Girl that Surrounds Me \\ Erika Puentes ${ }^{1}$ Mabel Edith Beltrán² Rafael Marroquín ${ }^{3}$
}

Para citar este artículo: Puentes, E.; Beltrán, M. E.; Marroquín, R. (2017). Subjetivación femenina: una mirada a la niña que me ronda. Infancias Imágenes, 16(1), 83-92.

\section{Resumen}

El presente artículo es producto de una exploración investigativa en curso, adelantada por el semillero de investigación Leidy Tabares (Leta), adscrito a la Licenciatura en Pedagogía Infantil de la Universidad Distrital Francisco José Caldas. Esta investigación pretende describir y analizar el proceso de configuración de las subjetividades de las licenciadas en formación durante su infancia, trabajado con un grupo poblacional que comprende estudiantes entre tercer y octavo nivel, desde el enfoque de género, a partir del relato de vida sobre sus prácticas de crianza o socialización, desde las cuales estas (mujeres hoy) fueron integradas o constituidas. Se trata de evidenciar las coordenadas discursivas (horizonte de sentido) que enrutaron (condujeron) su edificación subjetiva del hecho (histórico-sociocultural) de ser mujer, como también identificar su posición o ubicación particular frente al poder, la autoridad, la sociedad, la feminidad, la masculinidad, la sexualidad, el cuerpo, el conocimiento, etc.

Palabras clave: crianza del niño; formación de docentes; género; relato; enseñanza; educación de la primera infancia. [UNESCO Thesaurus http://vocabularies. unesco.org/browser/thesaurus/es/]
Recibido: 30-diciembre-2015 / Aprobado: 01-agosto-2016

\begin{abstract}
This article is the result of an ongoing research exploration, carried out by the research hotbed Leta (Leidy Tabares)—attached to the Curricular Project of the Bachelor's degree in Early Childhood Education offered by the University Francisco José Caldas. This research aims to describe and analyse the configuration process taking into account subjectivities of the graduates in training during their childhood, working with a population group of third and eighth grade students, from a gender perspective based on life stories about child-rearing and socialization practices, from which they (women nowadays) were integrated or incorporated. It is a question of demonstrating the discursive coordinates (horizon of sense) that routed (led) its subjective building of the (historical - sociocultural) fact of being a woman, as also identifying its position and/or particular place opposite to the power, the authority, the society, the femininity, the masculinity, the sexuality, the body, the knowledge, etc.
\end{abstract}

Keywords: child rearing; teacher education; gender; story telling; teaching; early childhood education.

\footnotetext{
* Artículo corto producto de la investigación en curso "Subjetivación femenina: prácticas de crianza de las licenciadas en Pedagogía Infantil UDFJC. Análisis desde un enfoque de género", iniciada en marzo de 2015, orientada por el docente Rafael Marroquín Fierro.

1 Estudiante de Licenciatura en Pedagogía Infantil, Universidad Distrital Francisco José de Caldas. Miembro del semillero de investigación Leta. Correo electrónico: erika.puentes01@gmail.com.

2 Estudiante de Licenciatura en Pedagogía Infantil, Universidad Distrital Francisco José de Caldas. Miembro del semillero de investigación Leta. Correo electrónico: politicasgenero.ud@gmail.com.

3 Magíster en Desarrollo Social y Educativo. Docente, coordinador del Proyecto Curricular de Pedagogía Infantil; director del semillero de investigación Leta. Correo electrónico: rmarroquin@udistrital.edu.co.
} 


\section{Introducción}

La teoría y los estudios de género han contribuido, a lo largo de muchas décadas, a investigar y analizar la constitución de las subjetividades e identidades femeninas y masculinas, develando o mostrando las diferencias existentes entre hombres y mujeres. Asimismo, los sentidos diversos que se le han atribuido, a través de la historia, a la feminidad y la masculinidad, denunciando con ello las disparidades e inequidades estructurales y puntuales de género que caracterizan a las sociedades tradicionales y contemporáneas.

En el actual documento se describe la configuración ${ }^{4}$ de la individuación infantil de las licenciadas en formación en pedagogía infantil de la Universidad Distrital Francisco José de Caldas, participantes en este estudio (en adelante LF), a la luz de las prácticas de crianza que experimentaron o vivenciaron en los contextos culturales y sociales de origen. Proceso de subjetivación cultural, social, político e históricamente situado, mediado y estructurado por las prácticas discursivas y no discursivas, las relaciones de poder (macro y micro) y las apuestas personales. Prácticas de crianza que ciertamente han reproducido la hegemonía patriarcal cuyo correlato se materializa en la desigualdad e injusticia de género, así como la exclusión secular de las mujeres.

Para dar cuenta de este propósito, en la primera parte del texto se explicitarán los presupuestos teóricos con los que se parte. Se detallarán los elementos conceptuales que sustentan y justifican la importancia de analizar la relación entre las prácticas de crianza y el proceso de subjetivación infantil de dichas mujeres. Además de ello, se explicará el sentido metodológico que se le dio a los relatos como herramienta de investigación y se argumentará por qué esta herramienta resultó apropiada para dar cuenta de los discursos de infancia y las prácticas de crianza descritas por las LF objeto-sujeto de esa exploración.

En el siguiente apartado se mostrará la ruta metodológica escogida y su efectiva aplicación. En la tercera y última parte se señalarán los hallazgos más relevantes y las conclusiones generales del trabajo.

\footnotetext{
4 Fenómeno entendido como un hecho social y político-histórico espacialmente situado.
}

Aquí el lector encontrará una breve descripción de los resultados provisionales más destacados, los cuales hacen referencia al proceso de fragilización a través del cual, o como efecto del cual, ha transitado o se ha configurado la subjetivación infantil de las licenciadas en cuestión; proceso evidente en las formas de enfrentar el poder, la autoridad y los conflictos, por ejemplo.

\section{Elementos conceptuales}

En las últimas décadas los estudios de género han tomado mayor relevancia, constituyéndose en el medio por excelencia para dar visibilidad a las condiciones de desigualdad social, económica, política y educativa que padecen las mujeres. No obstante, aún se siguen reproduciendo modelos de socialización sexistas, división de roles entre lo público y lo privado, donde a las niñas aún se les sigue formando implícitamente para el cuidado de otros, para la conformación de relaciones de parejas asimétricas, negando la poca o la nula presencia de su participación en la esfera pública, existiendo en la sociedad actual una serie de condiciones estructurales que fomentan la desigualdad y discriminación entre los géneros. Esto lo comprueba el índice de desarrollo humano, publicado por la Organización de las Naciones Unidas (ONU) citado en el artículo Los obstáculos de las mujeres en el periódico El Espectador (2015), pues —según este estudio-, se concluyó que los resultados con respecto a las mujeres son desalentadores en todos los aspectos.

Las prácticas de particularización femenina que se producen en la infancia de las licenciadas en formación no solo construye un tipo de feminidad fragilizada y subalternizada, sino que borra gran parte de la historia de cada mujer, dado el tipo de educación sexista a la que son expuestas en la infancia, que las homogeniza.

Partiendo de anterior, son necesarias investigaciones que aporten en la descripción y el análisis de estos procesos referidos a poblaciones concretas en situaciones específicas, tales como los concernientes a la configuración de las subjetividades de las docentes en formación durante su infancia. Para tal fin, es menester abordar y descodificar los contenidos discursivos y simbólicos de las prácticas de crianza, entendidas como dispositivos instituyentes 
de las identidades o subjetividades. Prácticas discursivas de crianza que modelan las interacciones cotidianas intersubjetivas, regulan las relaciones con los cuerpos, jerarquizan las relaciones niña/ adultos, configuran las representaciones e imaginarios de género, etc., lo cual hace que finalmente las mujeres sean de determinadas maneras.

En este sentido, resulta importante realizar unas precisiones conceptuales que definen desde nuestra perspectiva la razón de ser de las prácticas de crianza (prácticas discursivas) a la luz de los relatos de las propias LF, leídos desde el enfoque de género.

Prácticas de crianza: construcción de la subjetividad

Se entiende como prácticas de crianza toda aquella interacción y práctica lingüística o discursiva (gestual o no gestual) que realiza la persona (cuidador/a) que se encuentra a cargo de los niños y niñas, ya sea padre, madre abuela, tío o cualquier otro asignado o responsable de la educación, formación y atención del menor, que moldea pensamientos, imaginarios, acciones, actitudes, sentimientos, el cuerpo y la sexualidad infantil. Como lo nombra Rodríguez (2007), "toda familia socializa al niño de acuerdo a su particular modo de vida, el cual está influenciado por la realidad social, económica e histórica de la sociedad a la que pertenece" ( $p$. 93). En otras palabras, con estas prácticas de crianza se sigue reproduciendo la hegemonía patriarcal la cual se ha encargado de formar representaciones e imaginarios de género.

Las prácticas de crianza son entendidas como discurso y acción, por cuanto "constituyen un campo con reglas y relaciones que lo soportan, un campo que hace posible ciertos enunciados y no otros, enunciados que no representan una realidad previamente dada sino que la constituyen en esa práctica social" (Prada, 2010, p. 19).

Ciertamente, dichas prácticas de crianza siendo en sí mismas un discurso no se conciben como las causantes del tipo de organización social. Es más bien una relación bidireccional, pues si bien el discurso no es el que produce las inequidades de género y concepciones a cerca de la feminidad, masculinidad, entre otros, sí se constituye como una herramienta imprescindible para mantenerlos.
De esta manera, resulta pertinente mencionar a Boticelli (2011) en donde, retomando a Foucault, plantea el concepto de los dispositivos, configurados por prácticas discursivas y no discursivas, conforman y organizan la experiencia de acuerdo con un conjunto de condiciones preestablecidas, como es el caso de las docentes en formación de pedagogía infantil, quienes no han tenido otras posibilidades de experiencias, otras formas de ser mujer, que permitan realidades diferentes que pongan en entre dicho sus propias identidades y la razón de ser de las relaciones con los hombres. Botticelli, refiriéndose a Foucault en su texto sobre las prácticas discursivas, afirma:

[...] el sujeto no es otra cosa que la resultante de cierto tipo de dispositivos - aquellos involucrados en los diversos modos de subjetivación, la forma-sujeto se constituye en esos modos en los que el ser humano es objetivado desde una determinada relación de saber-poder que orienta prácticas particulares, en tanto elementos dinámicos, estas prácticas instauran los procedimientos y las técnicas mediante las cuales se elabora la relación de los sujetos consigo mismos y con los otros sujetos. (2011, p. 124).

Siendo el género la expresión de la división y distribución social de los y las sujetos, a quienes que se carga con estereotipos, significaciones y roles opuestos, efecto del paradigma biolgicista-esencialista, se justifican las diferencias de poder estableciendo un dominante y un dominado por mandato natural. Este ser dominante es el que define las concepciones del cuerpo, mentalidad, comportamientos, entre otros, de la vida del dominado (relación saber-poder). Pues, como lo nombra Rutter-Jersen (2012), quienes clasifican y definen generalmente son quienes tienen una posición económica y un estatus simbólico que le permite hacerlo. El problema radica en que estas concepciones acerca del género y los discursos que en consecuencia se imparten, generan exclusión, son legitimados y naturalizados desde la infancia a través de las prácticas de crianza, en donde se da forma a los niños de cierta manera y a las niñas de otra, respondiendo a las concepciones aprendidas $\mathrm{o}$ instituidas que se tienen sobre ellos. 
Es así como las prácticas de crianza se convierten en el habitus que envuelve o estructura sus vidas generación tras generación, reproduciendo patrones de desigualdad y antagonismo entre hombres y mujeres.

La niñez queda entonces circunscrita/atrapada en las condiciones y tradiciones culturales y sociales que determinan cierto tipo de contexto según la clase, el género y la raza, apropiando códigos y reglas que este les brinda, instaurando así el continuum de la reproducción cultural referida a los estereotipos, imaginarios, roles, relaciones, prácticas, etc., instituidos, donde las mujeres van a ser consideradas, tratadas, leídas, naturalizadas como delicadas, frágiles, vulnerables.

Las pautas de crianza, agenciadas especialmente por la familia - está como primer grupo de pertenencia y agente socializador de la infancia-, establecen reglas y prácticas discursivas que dan cuenta de cómo se debe dar forma, por un lado, a los niños y por el otro (de forma diferente) a las niñas. Se define que esta diferencia es el producto del orden natural/biológico entre hombres y mujeres, lo cual en realidad es una imposición sociopolítica y económica que se orquesta por medio de las creencias, las prácticas, los discursos, las reglas, los valores, etc., que fija las maneras de ser criados y establecerá los espacios, los límites de la inclusión social y las formas debidas de ser y estar dentro de la sociedad. En este sentido, dichas formas transversalizan las acciones cotidianas de los individuos impactando su subjetividad, asimilando y acomodando cada experiencia, aceptando de forma casi irrefutable lo que se ha enseñado por medio de las prácticas de crianza que se terminan por legitimar.

Por lo anterior, más que estudiar a las sujetos niñas, se debe hacer una detallada revisión de las prácticas de crianza o prácticas discursivas y no discursivas que se dan en dichos procesos. De allí la necesidad de involucrar la autobiografía de cada sujeto de estudio, ya que ubica minuciosamente lo que fue y es, esa construcción subjetiva configuradas a partir de dichas prácticas.

\section{Relato: un discurso en esencia}

El discurso es un modelo de subjetivación que es llevado a cabo en las instituciones como las familias y las escuelas: "los discursos carecen de autoría única por cuanto no emanan del interior de un sujeto, sino que articulan el conjunto de condiciones que permiten cierto orden de cosas" (Prada, 2010, p. 19). Los discursos, según Foucault (1978), constituyen prácticas sociales que producen y reproducen ideologías y formas de estar en el mundo. Es así como el discurso refleja y constituye prácticas e identidades, lo que sustenta además que las prácticas de crianza son un discurso operante que impacta la subjetividad.

El relato de infancia nos permite describir, leer o interpretar la realidad de los otros y la visión del mundo que los rodea. Es decir, "se interesa por el entendimiento del fenómeno social, desde la visión del actor" (Chárriez, 2012, p. 51). De igual manera, posibilita establecer tendencias entre un conjunto de relatos que hace que podamos abstraer una realidad general pues, como mencionan Santamarina y Marinas (1995) estas realidades están determinadas por las mismas comunidades y un periodo de tiempo concreto.

Los relatos autobiográficos permiten de esta forma analizar los discursos que imperan en ellas (LF), en los cuales se encuentra explícita o implícitamente las representaciones que cada una tiene acerca de sí misma como niña, a la luz del enfoque de género, visibilizando sus experiencias y percepciones de su propia subjetividad.

Para analizar el discurso que es expuesto en los relatos, y por ende los discursos empleados en las prácticas de crianza, el trabajo se apoyó en Foucault, entendiendo que dichos discursos son el producto de formas de actuar y pensar que a su vez estos se reproducen. De tal manera, se destaca que analizar los discursos permite entender cómo y por qué algo Ilega a considerarse como natural y legítimo.

Retomando a Iñiguez (2006), se entiende el análisis del discurso en tres dimensiones: el discurso ya sea en texto, el discurso como práctica que revela una situación social concreta y el discurso como práctica social. Este trabajo permite repensar la realidad inmediata que habitan las docentes en formación, esperando promover transformaciones tanto de sus discursos y acciones personales, como de sus prácticas profesionales en los espacios donde 
intervienen, pensando en modificar o resignificar dichas pautas de crianza a la hora de encarar in situ la formación de niños y niñas.

\section{Cómo se procede: metodología}

Este ejercicio de investigación se realiza en el marco del semillero de investigación Leta, a partir del interés de sus integrantes por analizar la configuración de las subjetividades infantiles de las licenciadas en formación, sobre el entendido que es en la niñez donde se estructura la individuación genérica a través y por medio de las prácticas de crianza como discurso y vivencia.

La investigación se enmarca en la orientación cualitativa, la cual permite aportar nuevas formas de comprender las prácticas sociales, entre ellas las prácticas educativas y de crianza. Es decir, desde el enfoque histórico hermenéutico se pretende categorizar las prácticas de crianza relacionadas con los roles de género, interpretar y explicar cómo estas han influido en la constitución de la subjetividad femenina promoviendo formas de ser, actuar y comportarse de las docentes en formación de la Licenciatura en Pedagogía Infantil, performatividad que en su mayoría las hace sumisas, subordinadas y con poca participación o presencia política y social.

Este estudio busca entonces caracterizar las prácticas de crianza con respecto al rol de género que configuraron la subjetividad infantil de las docentes en formación, por medio de la construcción e interpretación de los relatos de vida sobre sus infancias.

Los relatos nos permitieron evidenciar sus vivencias, eventos y hechos trascendentales, sus sensaciones, emociones, sentimientos y recuerdos que acompañan cada uno de "su ser niña".

El corpus documental con el que se trabajó corresponde a 42 relatos autobiográficos de estudiantes mujeres del proyecto curricular de Licenciatura de Pedagogía Infantil que se encontraban cursando los semestres de tercero a octavo, en el marco de la electiva, "Subjetividades de género", producidos en 2014.

Los relatos de infancia corresponden a las edades de 0 a 12 años, para lo cual se definieron unas pautas de elaboración determinadas que de manera breve se mencionan aquí. Se solicitó se explicitara el momento histórico en el que sucedió la infancia, las condiciones o circunstancias sociodemográficas de la familia, los ciclos o momentos más significativos en ese lapso de tiempo, la relación con el cuerpo, las relaciones de poder, conflictos y tensiones con los adultos del mismo sexo y el sexo opuesto, la vivencia escolar, así como los talentos y capacidades personales, y expectativas de infancia más destacados. Lo anterior, pensando en abordar globalmente la infancia y en desentrañar desde varios ámbitos los discursos que en la misma imperaban, y así determinar que ha intervenido en la configuración del sujeto niña que fue y aún se es. Para tal fin las estudiantes debían reconstruir la memoria e historia de este ciclo de vida interrogando a cuidadores(as), buscando detalladamente en álbumes de fotos, preguntando amigos(as), etc.

Tras los relatos en la investigación se procedió a describir los elementos más relevantes, para lo cual se diseñó una matriz que condensó la información. Esto nos permitió realizar una caracterización descriptiva de entrada en concordancia con los aspectos que se señalaron antes con el fin de identificar las prácticas de crianza con las que fueron prefiguradas sus subjetividades, relaciones, vínculos, apegos, emociones, representaciones de género, feminidad y masculinidad, etc.

Finalmente, se presenta un análisis reflexivo que da cuenta de este proceso — aún en construcciónel cual busca llamar la atención acerca de la importancia que tiene pensar el sujeto o los sujetos de la educación, agentes claves en la transformación de la escuela en Colombia, cuya dimensión se descuida u olvida a menudo en aras del cientificismo que impera en la Universidad; lo cual es imperativo cambiar, dado que aprehender la individuación subjetiva del maestro y la maestra (desde el enfoque de género) es pieza clave en esta dirección.

\section{Hallazgos iniciales}

A continuación, se dan a conocer los hallazgos que hasta el momento se han vislumbrado en este trabajo de investigación ${ }^{5}$.

\footnotetext{
5 En las citas textuales que tomaremos a continuación los nombres de las autoras de los relatos han sido cambiados con el fin de proteger sus identidades.
} 
Cabe mencionar que la caracterización poblacional de las sujetos-objetos de investigación, durante el periodo de la gestación hasta los 12 años, se encontraba en estratos socioeconómicos que no superan el 3, con una predominancia en el estrato 2 con $46 \%$, seguido del 3 con $31 \%$ y por el 1 con $23 \%$. El $87 \%$ de sujetos niñas vivieron su infancia en la ciudad de Bogotá. El 47 \% tuvo una tipología familiar nuclear, mientras el $38 \%$ extensa, el $9 \%$ monoparental y el $6 \%$ reintegrada.

El $76 \%$ cree en el dios judeocristiano mientras el otro porcentaje no arroja datos que puedan confirmar si forman parte de dicha creencia o no. Este alto porcentaje resulta ser un factor importante teniendo en cuenta las divisiones y roles de género y los valores morales y éticos que se manejan en cualquiera de las religiones que creen y profesan en dicho dios - ciertamente con algunas variaciones-.

Ahora bien, caracterizado el grupo poblacional retomaremos brevemente algunos de los hallazgos. A lo largo de los relatos se pudo identificar que el 88 grupo poblacional del que forman parte las LF era clase popular, y resulta pertinente nombrar que un $66 \%$ de las madres que se encontraban en una familia nuclear trabajaban, inicialmente por las condiciones económicas en las que la familia se encontraba, y quienes no lo hacían era por decisión de sus esposos:

A esta misma edad [7 años] más o menos mi mamá empezó a trabajar, pues las deudas primordialmente la cuota de la casa no daban espera, pero trabaja, porque actualmente lo sigue haciendo en la casa cuidando niños. (Jimena P.).

Mi mamá decidió retirarse de trabajar para hacerse cargo de mí mientras estaba más grandecita, entendiendo que ella no podía estar conmigo todo el día, pero cuando mi mamá decidió trabajar de nuevo mi papá no la dejó. (Carolina G.).

Fernández (2004) evidencia cómo históricamente la modernidad instauró un conjunto de dispositivos, prácticas, discursos y normas para subjetivizar y objetivizar a la mujer; diferencias marcadas por el género y la clase, donde las de clase media y alta, por ejemplo, debido a su patrimonio cultural, son acreedoras a una libertad controlada sin dejar de lado su papel como esposas y madres, y en las mujeres de sectores populares, su rol se reducía a velar por el cuidado de su marido, sus hijos y los hijos de las mujeres de clase media. Esto nos lleva a tocar otro punto importante en la configuración de la subjetividad femenina, como nos lo muestra Saltzman (1992) con la teoría de la estratificación de los sexos que se da con la naturalización de los roles o estereotipos que impone las instituciones en ese momento, un ejemplo de ello, es la división del trabajo: "[...] mi mamá no trabaja estaba encargada de las cosas del hogar y estar con mis hermanos, mi papá era el que sostenía la casa [...]" (Julieth M.).

Por otra parte, las mujeres que trabajaban en hogares de tipología extensa o monoparental lo hacían por la ausencia del padre o a que podían dejar a sus hijas al cuidado de sus tías o abuelas en su gran mayoría, pero escasamente a una figura masculina, denotando que el cuidado de las niñas se remite a las figuras femeninas. Aceptando así la división sexual del trabajo.

Viví un tiempo con mi mamá en el hogar Betania [...] después volvimos a casa a vivir con Mimí [abuela] y mi tía Camilita, fue lo mejor que pudimos hacer pues mi cuidado y crianza ahora estaban apoyada por ellas [...] mi mami empezó a trabajar. (Alexandra P.).

Lo anterior da cuenta del contrato sexual, como plantea Pateman (1995), que pone en evidencia la desigualdad que implica la división del mundo en dos espacios (público y privado), en la que vemos a las mujeres más tiempo con los niños y las niñas en casa. Indudablemente, esta es una visión que se da en la infancia y procesos no discursivos que se naturalizan y terminan reproduciéndose y enquistándose en las subjetividades.

En mis primeros años de vida, los pasé con mi mamá en la casa, ella cuidando de mí y de las cosas de la casa y mi papá trabajando para llevar el sustento de cada día. [...] Así que mi papá no veía la necesidad de que mi mamá trabajara y mi mamá tampoco sentía que tuviera que salir de ahí. (Angélica S.).

Estos casos de las niñas a cargo de las mujeres implican que desde pequeñas se vea una división 
sexual del trabajo y naturalizan que el cuidado se dé por parte de las mujeres. Esto incluso se puede leer a lo largo de casi todos los relatos en palabras como sus obligaciones, mi papá sí le ayudaba, nosotros le ayudábamos a mi mamá y que los proveedores económicos por naturaleza sean los hombres. Aunque en una cantidad considerable de familias nucleares trabajaba la madre es menester recordar que la tendencia era que esta situación se debía a la escasez económica.

Por algunos problemas mi papá se había ido de nuestro lado, pero volvió cuando cumplí cuatro años [...] esto llevó a que las cosas fueran diferentes, mi mamá ya no trabajaba y compartía más tiempo conmigo. (Luciana C.).

La escasez era mucha y el trabajo no abundaba, así que él [refiriéndose a su padre] no tuvo más opción que trabajar y trabajar para poder mantener a su esposa y a la nueva integrante de la familia. Ella [refiriéndose a su madre] mientras tanto se dedicaba a cuidar su embarazo, a cumplir con sus obligaciones de ama de casa y a tener listo todo. (Camila M.).

Por otro lado, se logró constatar que la infancia, en especial las sujetas niñas, se encuentra en "una sociedad que se caracteriza por privilegiar la autoridad del adulto. En muchas situaciones esto conlleva a que dicha autoridad utilice su lugar de poder, sometiéndolas y privándolas de la autonomía necesaria que les permitiría dejar de ser objetos" (Dibarboure, 2014, p. 14). Esto se encuentra sustentado y legitimado socialmente, ya que se considera a los hijos e hijas propiedad única y exclusiva de los padres, infancia que además debe rendir respeto a los adultos pues la misma es considerada como "menor" en todo el sentido de la palabra. Existe pues un falso significado de lo que es el respeto direccionando más a la obediencia, la sumisión y docilidad que posiciona en especial a las niñas (por la creencia tradicional de fragilidad), en un lugar de dependencia y efectivamente de vulnerabilidad.

Mis papás me guardaron en una burbuja por mucho tiempo, no les gustaba que saliera sola, que fuera a otras casas a jugar o a hacer algún trabajo, tenía horarios de llegada y de salida. (Tatiana L.).
A mi papá sí le tenía miedo, de hecho, la relación que mis hermanos y yo tuvimos con él cuando niños fue de total sometimiento. (Sofía B.).

Ella [refiriéndose a su abuela] fue también muy estricta conmigo y considero que en gran medida incidió en que yo fuera sumisa, ya que dentro de mi familia no sentí que tuviera voz y voto. (Ximena L.).

Mi mamá en varias ocasiones escogió la ropa que me compraba $[. .$.$] yo odiaba lo que me tocaba po-$ nerme, no sé a qué se debió, pero, aunque me pusiera brava, finalmente hacía lo que ella me señalaba, por lo que me acostumbré a ser obediente, no expresar mis ideas o sentimientos para no incomodar y por ende a guardarme las cosas. (Johana Z.).

En estos testimonios, se evidencia que las mujeres "participantes" le temen al conflicto, porque en la subjetivación de su infancia hay proceso de fragilización donde se les despolitiza y pierden autonomía. No actúan por ellas mismas, pues tienen un protector al lado, siendo este quien las defiende y cuida, por tanto, no están preparadas para combatir o luchar ya que otros lo hacen por ellas.

Por parte mía salía a defenderme mi hermana, mi mamá y mi papá y por parte de ella [su prima] salía mi tía Inés y mi tía Cecilia [...]. Nuestras peleas no eran de palabras solamente, nos jalábamos el pelo nos pellizcábamos y hasta aruñones nos dejábamos [...]. Yo siempre era más sumisa y al principio ella era la que me pegaba y yo no reaccionaba, sino que solo Iloraba. (Linda V.).

Esta fragilización hace que las niñas, ahora docentes en formación, consideren que la infancia necesita un protector, defensor de los peligros y mucho más si son niñas. Los relatos contenían fragmentos en donde se deduce que se sentían bien con la sobreprotección y el cuidado de sus padres (hombres) y sus hermanos. Por lo cual se acepta la idea de fragilización y vulnerabilidad de las niñas. Incluso no era necesario que les dijeran a ellas que corrían peligro (prácticas no discursivas), sino que la responsabilidad de que les sucediera algo estaba a cargo de otro, ni siquiera de sí misma. 
Salí con Cristian [hermano] a montar bici, la cicla se resbaló y caí en un barrizal rompiéndome la cabeza. Mi mami pegó un grito [...] a mi hermano lo regañaron fuertemente [...] y le dijeron que tenía que estar más pendiente. (Lorena F.).

Mi papá siempre me trataba con cuidado y procuraba evitar mis caídas. (Diana O.).

De igual manera, sus infancias se encontraron permeadas por prácticas discursivas que definían los roles e impartían inseguridad y vulnerabilidad a las niñas:

Yo no quería que nos devolviéramos a la casa, pero ya era tarde y mi abuelita decía que le tenía mucho miedo al centro pues se robaban a las niñas. (Sara U.).

Para mí en el colegio fue más fácil relacionarme con mujeres que con hombres, porque ellos me intimidaban. (Mery V.).

A medida que crecía, ellos [padres] decían que los riesgos eran inminentes y por eso me cuidaban, a veces en exceso para mi gusto. (Ingrid A.).

En síntesis, "la fragilidad femenina y la consecuente necesidad de protección masculina es también una construcción social e histórica" Fernández (2004) mediada por las prácticas discursivas y no discursivas.

La fragilización hace a las mujeres demandantes de afecto, y cuando no hay afecto Ilega el sentimiento de soledad y miedo, lo que crea el imaginario de incompletud y de la necesidad de una familia de tipo nuclear, en donde haya un padre protector y proveedor.

Yo lo extrañé mucho en mi infancia, siempre quise [...] poder tener un papá y una familia como todos mis demás primos(as), compañeros(as) y amigas del colegio. (Alejandra U.).

Me sentía triste, incompleta por no tener un papá que me recogiera a la salida del colegio y me protegiera en el camino. (Dayanna G.).

Desde la infancia las niñas han crecido con prácticas discursivas que legitiman la superioridad del hombre sobre la mujer, dejando de manifiesto una inequidad de género por considerar que las capacidades y alcances de cada cual son por esencia natural cuestiones que deben estar en el total pendiente de las LF, puesto que los discursos empleados, aunque son el resultado de una subjetivación de sí mismas, también siguen reproduciendo modelos de subjetivación en las aulas.

En educación física, donde influía la fuerza, la velocidad y muchos factores que, decía la profe, tenía más los hombres; y eso hacía que a nosotras nos mandaran a saltar lazo, a saltar la golosa, o cosas así, mientras ellos jugaban fútbol, corrían, etc. (Rocío P.).

Ver desde cortas edades las relaciones y roles de género las lleva a un proceso de identificación en un grupo de pertenencia (mujer-hombre, dominado-dominador, etc.) (Rutter-Jensen, 2012). Esto hace probablemente, desde su propia visión, tengan juicios sobre dichas interacciones, que al verse tan naturales en su infancia lleguen a considerar que así es el curso de la vida y asuman cierto rol (de mujer-madre, etc.). De esta manera no se le da la importancia que merecen ciertas situaciones y, además, en múltiples casos debido a la legitimación, dicha niña o mujer llegue a ser amiga, confidente, novia, esposa, etc., de su propio yugo y conviva, hasta donde la palabra logre llegar, con él.

Un día mi mamá llegó tarde a la casa [...] y mi papá le pegó de nuevo, en esta ocasión mi hermano ya con 9 años decidió enfrentarse a él [...]. Me acostumbre tanto que ya no le prestaba atención y así transcurrieron mis próximos años de niñez. (Paula J.).

Mi abuelo me enterraba con sus palabras, desde pequeña me repetía que yo no podía, que era una 'infame', buena para nada y más y más palabras hirientes que hicieron efecto en mi crecimiento; fuera de casa era muy tímida, callada, y sumisa [...]. A mi abuelo no le tenía odio, a pesar de su trato me sentaba con él a comer, le alcanzaba las onces o las comidas, le ayudaba a cuidar el ganado y le preguntaba sobre lo que leía [...]. (Gina T.).

Cuando sentíamos que llegaba de trabajar [refiriéndose a su padre] salíamos corriendo para los cuartos, los pasos del caballo en que se transportaba eran sonidos de ultratumba que de forma ilógica hoy 
todavía nos infunden temor al sentirlos en la esquina del corredor. (Ana N.).

Es así como el actuar diario de las niñas se encontró permeado por prácticas discursivas que coproducían inequidad, fragilización y vulnerabilidad. Las prácticas discursivas que se hacen no solamente les Ilega a las niñas, pues siempre los niños, hombres, están presentes en dichos discursos, lo que lleva a pensar, quizá, que si las niñas son vulnerables, los niños no, que si las niñas son vulnerables, quizá, es un hombre el que las vulnera.

Finalizando, cabe mencionar que de dicha manera las prácticas discursivas y no discursivas que forman parte de las prácticas de crianza, de manera conjunta con la organización social, coproducen realidades que hacen "sobrevivir" a las niñas y los niños en una sociedad patriarcal, violenta $y$ machista.

\section{Conclusiones previas}

La dependencia emocional, la escasa participación política, la sumisión, la subordinación y el subcapital cultural son hechos concretos que se presentan en las mujeres, en este caso las docentes en formación de la Licenciatura en Pedagogía Infantil de la Universidad Distrital Francisco José de Caldas "participantes en este estudio"; presentan comportamientos, maneras de ser y actuar que no solo reflejan la inequidad de género, sino que además, al ser parte de sus vidas personales y laborales, se replican y reproducen con las niñas y niños con quienes trabajan.

Los mencionados discursos que hacen parte de las prácticas de crianza construyen imaginarios de mujer, feminidad y masculinidad que contribuyen a una serie de imaginarios que han ido configurando sus identidades como mujer, y no se les ofrece elementos para desenvolverse en la esfera pública. Es decir, dicha dependencia les dificulta subjetivarse como sujetos de ciudadanía y derechos.

Es evidente que "existe un poder simbólico que tanto el dominado como el dominador comparten a partir de los valores y creencias aprendidas" en sus prácticas de crianza. Por lo tanto, el dominado hace legítimas y naturales las lógicas de dominación que, "sin saberlo ni quererlo reproduce, haciéndose partícipe del orden social imperante" (Dibarboure, 2014, p. 20).

A modo de cierre, se quiere nombrar que es importante trabajar estos aspectos con las docentes en formación para que se deje de legitimar las prácticas de crianza y relaciones con la infancia que sigan estatutos tradicionales acerca de la binariedad sexo/género, sus debidos comportamientos y formas de estar en el mundo. Pues, pensar las relaciones de género con un carácter biológico, natural, le otorga a esta definición un espacio incuestionable e inmutable. Como Finco (2015) menciona, esto implica no darse cuenta de que ese modo único y difundido de comprensión es reforzado por las instituciones sociales, como la familia y la escuela, y omiten que esas preferencias son construidas socialmente y, por tanto, están sujetas a modificaciones. Heller (1992, citado en Finco, 2015), señala el poder que tiene el sujeto para posibilitar transformar su realidad, siempre y cuando este se encuentre consciente de la misma y no parta de prejuicios "todo prejuicio impide la autonomía del ser humano, es decir, disminuye su relativa libertad ante el acto de elección, al deformar y, consecuentemente, estrechar el margen real del individuo" (p. 86).

De esta manera, la subjetividad de las niñas y la forma en la que se desenvuelven las prácticas de crianza serán fundamentales para su desarrollo como seres sociales y romper con la mirada acrítica que reproduce en el tiempo la inequidad de género, en donde se evidencia una injustificable supremacía de los hombres sobre las mujeres.

Para que las mujeres en general puedan ejercer su dimensión política y ciudadana como personas, llevando a cabo una vida libre y autónoma sin dependencias emocionales o económicas, se hace necesario implementar nuevas y diferentes prácticas de crianza dentro de las diferentes instituciones, logrando así una subjetividad basada en el ejercicio pleno de sus derechos.

Igualmente, se quiere resaltar que las sujeto-objeto han pasado por pequeños y grandes cambios acaecidos por múltiples y contradictorias circunstancias que se presentan a lo largo de sus vidas como mujeres, sin dejar de lado las tendencias que revelan aún, aspectos tradicionales, planteamos seguir adelante con la presente investigación 
—entendiendo que se encuentra en curso - sobre los pequeños y grandes cambios que han constituido la subjetividad femenina, proyectando la apuesta de entrever o develar los intersticios, señales o indicios que "reflejan" dichos cambios, modificaciones o resignificaciones de las identidades infantiles femeninas. Dado que el saber, el poder y los sujetos se encuentran situados en un momento histórico-social determinado, señalando además que hay una serie de matices diferencias o singularidades, según la época, la coyuntura, el contexto, los cambios y dinámicas sociopolíticas, económicas y culturales particulares que afectan o afectaron la construcción de estas identidades infantiles.

\section{Referencias}

Botticelli, S. (2011). Prácticas discursivas. El abordaje del discurso en el pensamiento de Michel Foucault. Instantes y Azares. Escrituras nietzscheanas, 9: 111-126. Recuperado de: http:// www.instantesyazares.com.ar/wp-content/ uploads/2016/12/Instantes-9.pdf

Chárriez, M. (2012). Historias de vida: una metodología de investigación cualitativa. Revista Griot, 5(1): 50-67.

Dibarboure, J. (2014) Análisis de las prácticas de crianza en familias que sufren violencia doméstica y sus posibles repercusiones en niños y niñas. (Tesis de grado). Universidad de la República, Montevideo.

Fernández, A. (2004). Historias de infancias. En: C. I. García (ed.), Hacerse mujeres, hacerse hombres: dispositivos pedagógicos de género. Bogotá: Siglo del Hombre, Universidad Central-DIUC.
Finco, D. (2015). Igualdad y género en las instituciones educativas de la primera infancia brasileña. Revista Latinoamericana de Ciencias Sociales, Niñez y Juventud, 13(1): 85-96.

Foucault, M. (1978). Arqueología del saber. Madrid: Siglo XXI.

Heller, A. (1992). O cotidiano e a história. São Paulo: Paz e Terra.

Iñiguez, L. (2006) Introducción. En: L. Iñiguez (ed.), Análisis del discurso: Manual para las Ciencias Sociales. Barcelona: UOC.

El Espectador. (18 de diciembre de 2015). Los obstáculos de las mujeres. Recuperado de: $\underline{\text { http:// }}$ www.elespectador.com/opinion/editorial/ los-obstaculos-de-mujeres-articulo-606509

Pateman, C. (1995). El contrato sexual. Barcelona: Anthropos.

Prada, N. (2010). Placeres peligrosos: discursos actuales sobre la sexualidad de las mujeres en el periódico El Tiempo. (Tesis de Maestría). Bogotá: Universidad Nacional de Colombia.

Rodríguez, A. (2007). Principales modelos de socialización familiar. Foro de Educación, 5(9): 91-97.

Rutter-Jersen, C. (2012). Temblores: notas sobre sexo, cultura y sociedad. Bogotá: Ediciones B.

Saltzman, J. (1992). Equidad y género: una teoría integrada de estabilidad y cambio. Madrid: Cátedra.

Santamarina, C.; Marinas, J. (1995). Historias de vida e historia oral. En: J. Delgado; J. Gutiérrez (eds.), Métodos y técnicas cualitativas de investigación en Ciencias Sociales (pp. 225-240). Madrid: Síntesis. 